\title{
Interactive comment on "Optimal Precursors Identification for North Atlantic Oscillation using CESM and CNOP Method" by Bin Mu et al.
}

\section{Anonymous Referee \#2}

Received and published: 10 November 2020

This manuscript describes the impact of the optimal precursor in modifying the North Atlantic Oscillation (NAO).

In this work, the authors compute the most efficient perturbation in a nonlinear context using a state-of-the-art climate model without the need for an adjoint. In this particular study, the method is applied to weekly NAO changes. This is interesting and show some potential. However, I did not find the paper well written and some of the diagnostics and figures are hard to follow.

I do not recommend this work for publication as it is. With some clarifications and a substantial amount of rewriting, I feel that this work could become publishable. 
The main issue of the study is a lack of focus and consistency. After reading the manuscript I am still uncertain on the scientific question(s) tackled here. I can mention three: (1) Perturbation of NAO (i.e., physics), (2) Development of PGAPSO with application to the CESM climate model (i.e., applied mathematics); and (3) Numerical performance of PGAPSO with CESM (numerical science). Of these three question, none was properly answered in the study. This is mostly due to the disparity of the three questions (and their topics) and that the authors tried to tackle them all in a single manuscript.

I would suggest the authors to choose and to focus on a single scientific question and to answer it in details.

Please see my more specific comments below.

Specific Comments:

1) Overall the abstract is not well written (see specific suggestions below). It is quite unclear what is the scientific question, how it will be tackle, and the conclusion of the work.

2) More generally the text is not well written. The English is quite poor and the citation seems to suffer from format problems. More fundamentally there is a lot of terminologies that are not defined (e.g., "NAO event", "Cases") or switch along the manuscript (e.g., CNOP, PGAPSO, OPR). This is not acceptable and extremely confusing.

3) The introduction suffers from the lack of contextualization from other "perturbation" method. For instance it would have been nice to acknowledge other method that has considered change in atmospheric dynamics (SVD). It also suffer from the lack of introduction of fundamental concept mentioned and used in the study. For instance, the predictability of the NAO is not discussed in depth. The concept of predictability is not properly mentioned, whereas it is crucial for the study.

Printer-friendly version

4) The experimental set up does not make sense at all. . I do not understand why the 
perturbation is restricted to the Polar region (north of $60 \mathrm{~N}$ ). What scientific question can be answered here? I feel that this choice is quite random and does not follow from NPGD the vast literature on the topic (or at least it is not motivated that way).

6) Overall there is a lack of consistency and some information are missing making the manuscript hard (if not impossible) to follow. This also highly negatively impacts the reproducibility of the study.

Minor/Technical Comments:

p.1-I.1: replace "seesaw phenomenon" by "variability". I have never seen the term "seesaw" - which implicitly implies an interplay between two regions - associated to the NAO. But I might be wrong.

p1.-I.2: replace "has a profound influence" on by "influences".

p.1-I.1: replace "for" by "of"

p.1-I.3: "NAO event" is not defined yet.

p.1-I.1: replace "the NAO anomaly pattern" by "NAO anomaly"

p.1-I.15: remove "phase reversing" and "in the meridional direction"

p.1-I.16: replace "is mainly" by "can be"

p.1-I.18: replace "mode of atmospheric circulation variability" by "variability mode of the atmospheric circulation"

p.1-I.22: replace "quantified" by "quantitative"

p.1-I.22: replace "difference between normalized SLP" by "normalized difference between SLP"

Printer-friendly version

p.1-I.24: add "over" before "Azores"

Discussion paper

p.1-I.24: the term "turbulence" does not make sense here. 
p.2-I.2: replace "etc." by "for instance"

p.2-I.2: Please clarify the location of the surface temperature variation mentioned.

NPGD

p.2-I.3: "The NAO can be regarded as a nonlinear initial value problem" is quite out of context, please clarify or introduce it more specifically.

p.2-I.4: "NAO events" is not define and the association of "NAO" and "event" does not make sense. You define the NAO as variability. What is an event? An extreme value of the variability?

p.2-I.20: The term "gradually" does not make sense.

p.2-I.22: remove the "Jiang et al" in the bracket.

p.2-I.22: remove the "Dai et al" in the bracket.

p.2-I.28: remove the "Marshall and Molteni" in the bracket.

p.2-I.30: remove the "Jiang et al" in the bracket.

p.2-I.30: remove the "Dai et al" in the bracket.

p.2-I.29: the term "optimally growing initial error (OGE)" is hard to follow without further explanation

p.3-I.3-5: This sentence is a strong statement. References are need here.

p.4-I.21: replace "between $60 \mathrm{~N}$ and $90 \mathrm{~N}$ " by "north of $60 \mathrm{~N}$ "

p.5-Fig.2: "The region of the NAO". The NAO is not defined by a region. This does not make any sense.

p.6.-I.1: Please add a reference for "linear singular vector".

Printer-friendly version

p.6-14-5: The predictability of the NAO was not introduce at all in the intro. The "predictability" concept (and references) neither...

Interactive

comment 
p.6-I.6: "NAO events" is still not defined. At this stage of the manuscript a quantitative definition is needed.

NPGD

p.6-I.10: It is unusual to write the S_0 inside the bracket. Also, with this notation, it looks like the operator ( $\mathrm{M}$, which is a function of $\mathrm{S} 0$ ) is equal to the vector (St).

p.6-I.14: Most term are undefined (S', \deltaS).

Interactive

comment

p.6-I16: "NAOI" is not defined.

p.6-I.18-19: "NAOI(NAO+) and NAOI(NAO-)" are not defined and do not make sense.

p.7-I.2: $D$ is not defined

p.7-I.3: $10 \%$ of the local variation? Would that not be more usual to take even something lower for predictability such as $1 \%$. Also using a typical variation in time rather than space would be more ideal.

p.7-I.8: Remove "Liu" in the bracket

p.7-Fig.3: "North Atlantic Region [...]" Are you suggesting that your EOF was computed on this restricted region? If so please clarify,

p.8-I.18: Use $a$ ' in the right hand side of the equation, if it is a new variable (i.e., anomaly).

p.9-I.2: Be more quantitative on how smaller the space is.

p.15-Fig.8: How is it built? Is it a composite of NAO+ and NAO- from the 50 iterations of the distribution depicted in Fig.7? Please clarify?

p.15-I.9: I don't understand how the random procedure work? Is it a composite of random perturbation leading to + and - NAO values? If so how many random perturbation were used to build the composite? If the composite is built as a mean of an ensemble, the figure should show the standard deviation to depict the level of uncertainty?

Printer-friendly version

p.15-I.12-13: I did not find the definition of the cases?

Discussion paper

C5 
p.16-Fig.9: The title should go on top, the $x$-axis should be labelled.

p.16-I.11-12: For CNOP_NAO+ and CNOP_NAO-, I guess? Please clarify.

NPGD

p.17-Fig.10: If I understand correctly the random method (i.e., composite/mean of - and + outcomes) the pattern should be symmetric by definition, isn't it? Here it is definitely not! Is it a mistake? Please clarify? Also, replace PGAPSO by CNOP for consistency (and be consistent in all figures and all along the text).

p.18-Fig.12: Add "for the CNOP_NAO+ and CNOP_NAO-“" at the end of the caption

p.18-I.17-19: I don't understand that. . .

p.19-Fig.13: Replace "OPRs" by "CNOPs" in the caption, because you computed other types of "OPRs" (i.e., random and BV). Also be consistent throughout the manuscript (figure and text).

p.20-Fig.15: Which case this figure refer to? Is it the response (on temperature?) of temperature only perturbation? I don't understand the point here... There is a huge lack of information here (and elsewhere), making the reproducibility impossible and making the manuscript extremely hard to follow (if not impossible).

p.23-I24-26: Improving the CESM computation is not without interest, but I don't understand how it fits with the particular target here: CNOP. It would have been more interesting to show how the computation of CNOP can be improved for "constant-performance" of CESM, isn't it? I may be missing the point, but it does not seem align with the rest of the analysis.

Interactive comment on Nonlin. Processes Geophys. Discuss., https://doi.org/10.5194/npg2020-27, 2020.

Printer-friendly version

Discussion paper
Interactive

comment 